María Augusta Gutiérrez-Sarmiento; Cecilia Ivonne Narváez-Zurita; Jorge Edwin Ormaza-Andrade

DOI 10.35381/cm.v6i2.381

\title{
Control interno permanente en la administración de los inventarios del Hospital Homero Castanier Crespo
}

Internal and permanent control in the administration of the inventories of the Hospital Homero Castanier Crespo

\author{
María Augusta Gutiérrez-Sarmiento \\ maria.gutierrez78@est.ucacue.edu.ec \\ Universidad Católica de Cuenca, Cuenca \\ Ecuador \\ https://orcid.org/0000-0002-7806-4632 \\ Cecilia Ivonne Narváez-Zurita \\ inarvaez@ucacue.edu.ec \\ Universidad Católica de Cuenca, Cuenca \\ Ecuador \\ https://orcid.org/0000-0002-7437-9880 \\ Jorge Edwin Ormaza-Andrade \\ jormaza@ucacue.edu.ec \\ Universidad Católica de Cuenca \\ Ecuador \\ https://orcid.org/0000-0001-5449-1042
}

Recibido: 05 de agosto de 2020

Aprobado: 01 de octubre de 2020 


\title{
RESUMEN
}

La investigación estuvo orientada a la evaluación del sistema de control interno en el hospital Homero Castanier Crespo de la ciudad de Azogues, con la utilización de los elementos ambiente de control, evaluación de riesgos, actividades de control, información y supervisión, contemplados en el informe coSO I. En este contexto, el objetivo del estudio es establecer los componentes que inciden en el procedimiento de evaluación del sistema de control interno de inventarios de la institución pública de salud. El artículo tuvo un abordaje no experimental, con alcance descriptivo; la información se obtuvo mediante revisión teórica y encuestas, éstas proporcionaron un mejor entendimiento sobre los procesos que se llevan a cabo en la entidad con relación a la administración de inventarios; los resultados muestran que existe una carencia sistemática en la valuación del control interno de inventarios que afecta la eficiencia del servicio y la fiabilidad financiera.

Descriptores: Financiación; tributación; empresa; crecimiento económico; planificación estratégica. (Palabras tomadas dl Tesauro UNESCO).

\begin{abstract}
The research was aimed at evaluating the internal control system in the Homero Castanier Crespo hospital in the city of Azogues, with the use of the elements of control environment, risk assessment, control activities, information and supervision, contemplated in the report COSO I. In this context, the objective of the study is to establish the components that affect the evaluation procedure of the internal inventory control system of the public health institution. The article had a non-experimental approach, with a descriptive scope; The information was obtained through theoretical review and surveys, these provided a better understanding of the processes that are carried out in the entity in relation to inventory management; The results show that there is a systematic lack in the internal inventory control valuation that affects the efficiency of the service and financial reliability.
\end{abstract}

Descriptors: Financing; taxation; enterprises; economic growth; strategic planning. (Words taken from the UNESCO thesaurus). 


\section{INTRODUCCIÓN}

Los inventarios mantienen los productos, materias primas, insumos, envases y otros elementos necesarios para la producción o prestación de un servicio determinado, en este rubro se maneja buena parte del capital de una institución, que debe administrarse con cuidado, pues los procesos dependen del abastecimiento requerido para un trabajo eficiente, por consiguiente, la administración de inventarios debe considerar aspectos como el número de unidades necesarias para la producción o servicio, el tiempo de elaboración del inventario, la adquisición de artículos de prioridad y los cambios en los costos de los artículos del inventario.

El control interno se define como el grupo de procedimientos para observar el cumplimiento de los departamentos y cotejarlos con los datos reportados, es así que, un control adecuado se convierte en la reducción de costos, por consiguiente, el descubrimiento de fraudes, daños o robos, evita pérdidas (Juca, Narváez, Erazo, \& Luna, 2019). Los elementos del control interno tienen por finalidad analizar el ambiente de control, la evaluación de los riesgos, el control de actividades para prevenir riesgos, la información y comunicación y la supervisión.

En este orden de ideas, el control interno de inventarios presenta dos enfoques, por un lado, el control operativo encargado de la determinación de las existencias en un grado aceptable, y por otro, el control contable que establece el mantenimiento de los registros de forma técnica (Ortiz, Narváez, \& Erazo, 2019).

En este contexto, en el hospital Homero Castanier Crespo, se ha detectado un sistema deficiente de control debido a un control interno poco sistemático y que genera dificultades a los funcionarios que prestan el servicio, por consiguiente, la presente investigación pretende responder a la problemática: ¿cómo incide el sistema de control interno de los inventarios en el hospital Homero Castanier Crespo?, en efecto, la investigación tiene como objetivo establecer un procedimiento de evaluación del sistema 
de control interno en el hospital Homero Castanier Crespo para la determinación de la situación actual de los inventarios.

\section{Referencial teórico}

\section{Inventarios: tipos, contabilización y valuación}

Los inventarios se definen como el conjunto de productos, materiales $u$ otros artículos almacenados para la venta, distribución, consumo o proceso de transformación, dependiendo la finalidad de la industria o empresa (Durán, 2012). Representan una de las inversiones más importantes de acuerdo con el resto de los activos, son primordiales para optimizar las utilidades, aumentar las ventas, mantener activo el proceso de producción y servicios de atención al cliente.

El inventario resulta relevante en industrias y empresas, pues tienen el propósito de satisfacer la demanda de los consumidores sin tener un tiempo excesivo de espera 0 para que el proceso productivo no se vea interrumpido frente a la falta de productos. Se considera como un instrumento regulador que mantiene el equilibrio entre los flujos reales de entrada y salida (García, 2017).

Dependiendo de los artículos que se desea contabilizar existe una gran variedad de inventarios, los que poseen diferentes objetivos concretos.

1. Inventario de materias primas: está compuesto por materiales con los que se elaboran los productos que aún no han sido procesados.

2. Inventario de productos en proceso de fabricación: lo componen los bienes comprados por las industrias. Se cuantifica de acuerdo a la cantidad de materiales, gastos de fabricación y mano de obra.

3. Inventario de productos terminados: son aquellos que se transforman con el propósito de ser comercializados como productos elaborados.

4. Inventario de suministros de fábrica: en este grupo se encuentran los materiales con los que se elaboran los productos, los que no se cuantifican con exactitud. 
CIENCIAMATRIA

Revista Interdisciplinaria de Humanidades, Educación, Ciencia y Tecnología

Año VI. Vol. VI. N². Edición Especial II. 2020

Hecho el depósito de ley: pp201602FA4721

ISSN-L: 2542-3029; ISSN: 2610-802X

Universidad Nacional Experimental Francisco de Miranda (UNEFM). Santa Ana de Coro. Venezuela

María Augusta Gutiérrez-Sarmiento; Cecilia Ivonne Narváez-Zurita; Jorge Edwin Ormaza-Andrade

Una clasificación más completa de los inventarios se presenta en la tabla 1.

Tabla 1.

Tipos de inventarios.

\begin{tabular}{|c|c|c|c|c|c|}
\hline \multicolumn{6}{|c|}{ Tipos de inventarios según: } \\
\hline El momento & El periodo & La forma & La función & La logística & Otros \\
\hline \multirow[t]{2}{*}{$\begin{array}{l}\text { Inventario } \\
\text { inicial }\end{array}$} & $\begin{array}{l}\text { Inventario } \\
\text { discontinuo }\end{array}$ & $\begin{array}{l}\text { Inventario de } \\
\text { materias primas }\end{array}$ & $\begin{array}{l}\text { Inventario en } \\
\text { tránsito }\end{array}$ & $\begin{array}{l}\text { Inventario de } \\
\text { existencias } \\
\text { para } \\
\text { especulación }\end{array}$ & $\begin{array}{l}\text { Inventario } \\
\text { físico }\end{array}$ \\
\hline & $\begin{array}{l}\text { Inventario } \\
\text { perpetuo }\end{array}$ & $\begin{array}{l}\text { Inventario de } \\
\text { productos en } \\
\text { fabricación }\end{array}$ & $\begin{array}{l}\text { Inventario de } \\
\text { ciclo }\end{array}$ & $\begin{array}{l}\text { Inventario de } \\
\text { existencias } \\
\text { obsoletas }\end{array}$ & $\begin{array}{l}\text { Inventario } \\
\text { mínimo }\end{array}$ \\
\hline \multirow{3}{*}{$\begin{array}{l}\text { Inventario } \\
\text { final }\end{array}$} & & $\begin{array}{l}\text { Inventario de } \\
\text { productos } \\
\text { terminados }\end{array}$ & $\begin{array}{l}\text { Inventario de } \\
\text { seguridad }\end{array}$ & $\begin{array}{l}\text { Inventario en } \\
\text { ductos }\end{array}$ & $\begin{array}{l}\text { Inventario } \\
\text { máximo }\end{array}$ \\
\hline & & $\begin{array}{l}\text { Inventario de } \\
\text { suministros de } \\
\text { fábrica }\end{array}$ & $\begin{array}{l}\text { Inventario de } \\
\text { previsión }\end{array}$ & $\begin{array}{l}\text { Inventario de } \\
\text { existencias de } \\
\text { seguridad }\end{array}$ & $\begin{array}{l}\text { Inventario } \\
\text { disponible }\end{array}$ \\
\hline & & $\begin{array}{l}\text { Inventario de } \\
\text { mercancías }\end{array}$ & $\begin{array}{l}\text { Inventario de } \\
\text { desencaje }\end{array}$ & $\begin{array}{l}\text { Inventario de } \\
\text { existencias } \\
\text { naturaleza } \\
\text { regular }\end{array}$ & $\begin{array}{l}\text { Inventario en } \\
\text { línea }\end{array}$ \\
\hline
\end{tabular}

Fuente: Caurin (2017).

En este contexto, el control del inventario desempeña un rol importante en los planes de cualquier empresa, puesto que permite mantener un control acerca de los productos 0 suministros almacenados en las bodegas (Ávila, 2010), así como su proceso de tránsito, desde la llegada hasta la salida. Cabe mencionar, que el control de inventarios tiene la capacidad de predecir, es decir, fija un cronograma de producción, para conocer la cantidad de artículos o materia prima que se procesa en un determinado período, para lo cual debe existir un balance entre lo prescrito en el inventario y lo utilizado.

Es más, realizar un control de inestabilidad de los suministros, permite garantizar la provisión permanente en el caso de existir una falta de seguridad por los proveedores o 
María Augusta Gutiérrez-Sarmiento; Cecilia Ivonne Narváez-Zurita; Jorge Edwin Ormaza-Andrade

si quedan pocas unidades de un determinado artículo en bodega. Con una apropiada adquisición de acuerdo a la cantidad y evitar el impacto de la inflación de los costos a mediano o largo plazo y la posibilidad de ofrecer descuentos a los clientes sin perder el margen de utilidad, sobre todo en ocasiones especiales (García, 2017).

Por otro lado, un adecuado inventario mantiene la administración correcta de las existencias en las bodegas y evita la salida sin control de los productos, como en el caso de las empresas estatales, donde son comunes los problemas de falta de mercadería o artículos cuando se realiza una intervención contable a sus cuentas y bodegas, al inicio de un nuevo periodo fiscal, cuando se realizan controles contables de rutina o al momento de la designación de nuevas autoridades, por ello, es necesario contar con un sistema de contabilización efectivo y la utilización de métodos de evaluación de inventarios de acuerdo a la actividad a realizarse (Hurtado, 2014).

Al respecto, los sistemas de contabilización se utilizan para registrar la cantidad de mercadería existente, así como permiten establecer el costo de la mercancía vendida. En este sentido, Durán (2012) expresa que existen dos métodos o sistemas básicos de control de inventarios:

1. Sistema de inventario periódico: este control se realiza cada determinado tiempo o período y es necesario ejecutar un conteo físico de las existencias que se tienen a disposición.

2. Sistema de inventario permanente o perpetuo: este control mantiene un registro continuo de cada artículo del inventario, adicional los registros que exponen la mercadería disponible en todo momento.

Ahora bien, los métodos de valuación de inventarios son técnicas utilizadas para seleccionar y aplicar una base específica para evaluar los inventarios en términos monetarios, por lo tanto, es un proceso vital cuando los precios unitarios de compra son diferentes. Entre los métodos más utilizados se tiene: 
María Augusta Gutiérrez-Sarmiento; Cecilia Ivonne Narváez-Zurita; Jorge Edwin Ormaza-Andrade

1. Método FIFO o PEPS: se basa en que lo primero que entra, es lo primero en salir. Emplea una valoración basada en costos más recientes y la apreciación se adapta más a la realidad del mercado.

2. Método del Promedio Armónico o Ponderado: consiste en el promedio que se calculará ponderando los precios con las unidades adquiridas, luego divide los valores totales entre el total de las unidades (Fuertes, 2015).

\section{Control de inventarios hospitalarios}

Es indiscutible que los centros hospitalarios constituyen uno de los elementos fundamentales en el sistema de salud, en la cadena de atención a los usuarios o pacientes para suministrar insumos necesarios en el tiempo adecuado; por lo expuesto, se requiere la aplicación de diferentes herramientas diseñadas para el correcto manejo de los inventarios con la finalidad de minimizar los faltantes, rupturas y deshechos de stock, que afectan el servicio y la economía de la institución y la atención de los ciudadanos (Organización Mundial de la Salud, 2012).

El hecho de tener los datos del inventario en tiempo real, permite conocer con precisión cuándo y en qué cantidades se deben reponer los inventarios en las diferentes áreas del hospital, con el objetivo de no mantener gran inventario en cada sector, sino al contrario, tener la mayor cantidad concentrada en la bodega. Asimismo, sin distinción del servicio que brindan o del producto que proporcionan, se entiende que los inventarios representan una inversión considerable porque los niveles de existencia son altos al devengar costos asociados al almacenaje, operación, gestión, disposición y su control es imprescindible (Cardona, Orejuela, \& Rojas, 2018).

De acuerdo con la Organización Mundial de la Salud (OMS), el inventario de un hospital debe contener la información relevante a equipos médicos, accesorios, elementos de mantenimiento y oficina, equipos de seguridad, registro de materiales y residuos radioactivos, material fungible, entre los más importantes. Para realizar el informe 
adecuado de todos los elementos, es necesario incluir una serie de datos mínimos solicitados, como el número, tipo y descripción del artículo, el número de serie, modelo, fabricante, estado y la ubicación física en el hospital, en general las características físicas, el control de ingreso y egreso de los mismos (Organización Mundial de la Salud, 2012). Entre los principales pasos a seguir para la adecuada gestión de los inventarios en un centro hospitalario, resaltan los siguientes:

1. Recopilación inicial de datos por parte de una persona o equipo de personas en la que se realiza el inventario de todo el material del centro.

2. Actualización de la información cada vez que se realice una compra, se use, se cambie o se dé la baja de un equipo o cualquier insumo.

3. Revisión anual para comprobarse que la información del inventario es correcta y coincide con los registros contables.

\section{Control interno}

Se define como un proceso ejecutado por las autoridades de una institución y profesionales especializados para proporcionar información sobre la consecución de objetivos organizacionales vinculados a la eficacia y eficiencia de las acciones en la entidad, fiabilidad de la información contable y financiera en apego a las normas y leyes (López \& Pesántez, 2017). También se refiere a los modelos de comportamiento de la organización y la interiorización de las personas sobre la importancia que tiene el control para optimizar la institución, tomando en cuenta los valores con los que se trabaja, la capacidad de los funcionarios, la filosofía y las estructuras de actuación y servicio que se manejan (Ortega, Padilla, Torres, \& Ruz, 2017).

Entre los elementos que se pretenden supervisar con los controles están las decisiones equivocadas, el vínculo entre costos y beneficios de compras o errores humanos (Moscoso, 2015). Con base en lo expuesto, Se afirma que el control interno es una guía que se encuentra basada en los planes de las instituciones y todas las coordinaciones de 
María Augusta Gutiérrez-Sarmiento; Cecilia Ivonne Narváez-Zurita; Jorge Edwin Ormaza-Andrade

técnicas o métodos, dicho de otra forma, representa la defensa en los activos para confirmar una certeza de los informes contables, financieros o de administración (Camacho Villota, Gil Espinoza, \& Paredes Tobar, 2017).

La evaluación del control interno en las entidades, parte de un control efectivo de la información generada en los registros contables que aportan a la institución en la toma de decisiones administrativas adecuadas (Rincón, Grajales, \& Zamorano, 2012). Se pueda notar la relación entre los objetivos de la institución, la eficiencia en los registros contables que determinan una adecuada valoración de control interno para la consecución de los logros planificados con anterioridad (Chávez \& Vallejo, 2017). Al respecto, Quinaluisa, Ponce, Muñoz y Ortega (2018) precisan que los sistemas de control más utilizados por organismos internacionales son:

1. Coso (Committee of Sponsoring Organizations of the Treadway Commission, Estados Unidos): es un proceso que tiene por finalidad proporcionar un grado de seguridad razonable en cuanto a la consecución de objetivos como la eficacia y eficiencia de las operaciones, confiabilidad de la información financiera, cumplimiento de las leyes, reglamentos y normas que sean aplicables.

2. Coco (Criteria of Control, Canadá): define el control interno de una forma similar al modelo COSO, con la diferencia que este modelo provee un marco de referencia por medio de 20 criterios agrupados en 4 componentes, que se aplican para diseñar, desarrollar, modificar o evaluar el control interno.

3. Cadbury (Reino Unido): está orientado a proporcionar una razonable seguridad de efectividad de las operaciones, los elementos clave del modelo son esenciales y similares al modelo COSO, excepto la consideración de los sistemas de información integrados en los otros componentes y un mayor énfasis respecto a riesgos. 
Universidad Nacional Experimental Francisco de Miranda (UNEFM). Santa Ana de Coro. Venezuela

María Augusta Gutiérrez-Sarmiento; Cecilia Ivonne Narváez-Zurita; Jorge Edwin Ormaza-Andrade

4. SAC (Systems Auditability and Control): se basa en un modelo de metadatos que permite una integración semántica de control de acceso y una infraestructura de acreditación externa. Representa una solución al problema del control de acceso para entornos distribuidos, dinámicos y heterogéneos con base a la información semántica para conseguir que se consideren las propiedades particulares de los recursos accedidos.

A partir de lo expuesto, una de las normas más utilizadas para cumplir con las metas de control interno apegado a los estándares internacionales como las NIIF y los sistemas de control aceptados en el medio es el modelo COSO l; el mismo se utiliza por su versatilidad y adecuación a las necesidades de las instituciones (Pazmiño, Narváez, \& Erazo, 2019), cuenta con cinco componentes relacionados entre sí y están integrados en el proceso de dirección. Los componentes que se presentan son independientes del tamaño o naturaleza de la organización, de acuerdo con Pazmiño, Narváez y Erazo (2019) se identifican de la siguiente manera:

1. Ambiente de control: es el establecimiento de un ambiente en el cual se estimula las actividades del personal de la empresa con respecto al control de sus actividades, de acuerdo con la organización, comunicación, responsabilidad y desarrollo de valores y creencias.

2. Evaluación de riesgos: en este punto es necesario identificar y analizar los riesgos más relevantes para conseguir los objetivos planteados y la base para determinar la manera en que los riesgos deben ser mejorados. De la misma forma, denota los mecanismos necesarios para identificar y manejar ciertos riesgos asociados con los cambios, tanto los que influyen en el interior de la organización como en su entorno.

3. Actividades de control: son aquellas que realiza la administración central de la empresa para ejecutar a diario las actividades establecidas, ya sea de manera 
María Augusta Gutiérrez-Sarmiento; Cecilia Ivonne Narváez-Zurita; Jorge Edwin Ormaza-Andrade

manual o computarizada. Están determinadas de acuerdo a las políticas, sistemas y procedimientos y su importancia radica no solo en la forma correcta de hacer las tareas, sino en el aseguramiento del logro de los resultados.

4. Información y comunicación: identifica, recopila y comunica toda información relacionada con las actividades realizadas, de tal modo que, permite cumplir a cada trabajador con sus responsabilidades encomendadas. Es parte fundamental en la toma de decisiones, así como, en el seguimiento de las operaciones.

5. Supervisión y seguimiento: a partir de la ejecución del control interno, la administración debe realizar la revisión y evaluación sistemática que compruebe que se mantiene el adecuado funcionamiento del sistema a lo largo de un período de tiempo establecido, por medio de supervisiones continuas, evaluaciones periódicas o una combinación de ambas.

Finalmente, el control interno en el área de inventarios se interpreta como una forma clara de mantener los procesos y los estados contables de un departamento del que dependen los usuarios y las personas relacionadas con el servicio que se ofrece. Para ello, es necesario una planificación del control interno a ejecutarse, que incluya el personal idóneo para las tareas, las estrategias y las técnicas adecuadas de control, con la distinción de etapas y la ejecución de las mismas con la organización debida.

\section{Métodos de evaluación de control interno aplicado a inventarios}

Para realizar una evaluación de control interno existen tres métodos como son el descriptivo, el de cuestionarios y el método gráfico, los mismos permiten efectuar un trabajo de manera adecuada y efectiva.

1. Método descriptivo: radica en la explicación por escrito de los procedimientos para la ejecución de las distintas operaciones específicas de control interno en el 
María Augusta Gutiérrez-Sarmiento; Cecilia Ivonne Narváez-Zurita; Jorge Edwin Ormaza-Andrade

departamento de inventarios, realizando una formulación de memorandos en los cuales se transcribe en forma expresiva los distintos pasos del control operativo.

2. Método de cuestionarios: para este procedimiento previamente hay que establecer una relación de preguntas sobre aspectos básicos de la evaluación de control a realizar y a continuación se procede a la obtención de las respuestas para averiguar las debilidades del control interno.

3. Método gráfico: este método se basa en la esquematización de las operaciones de control por medio de la utilización de gráficos como: cuadros, dibujos, figuras geométricas, entre otros, los mismos que representan archivos, zonas, secciones o ubicaciones en el departamento que indican y explican el flujo de las actividades que se realizan en ese sector (Velasquez, 2011).

Es importante destacar que, al momento de elaborar un sistema de evaluación de control interno, resulta conveniente la utilización de los tres métodos antes señalados a razón de que permiten obtener de manera óptima la información relevante a las actividades y las existencias de los inventarios y, por consiguiente, los resultados son más favorables. Sobre todo, cuando se realiza de manera periódica, cuando la situación lo amerite o de acuerdo a las circunstancias este debe ejecutarse de forma mensual, semestral o anual. El control interno en el caso de los inventarios tiende a realizarse a tres grandes grupos en los cuales se hallan las actividades o tareas específicas que realiza la evaluación:

1. Ingreso de existencias: el jefe o encargado del departamento de inventarios debe conocer la fecha y hora aproximada de las mercaderías o insumos que se han solicitado de forma previa y que serán almacenadas en ese lugar. Para ello debe contar con la documentación en regla que detalla los datos referentes al o los artículos o insumos solicitados como son la cantidad, el precio, tipo, fecha de caducidad (en el caso que lo tenga), la persona que autorizo la compra y los datos del proveedor. Adicional, deberá realizar el conteo manual de la mercadería que 
Universidad Nacional Experimental Francisco de Miranda (UNEFM). Santa Ana de Coro. Venezuela

María Augusta Gutiérrez-Sarmiento; Cecilia Ivonne Narváez-Zurita; Jorge Edwin Ormaza-Andrade

ingresa y comparar su estado físico y si cumple con los requerimientos especificados en la factura y la orden de compra, al final dispondrá de un informe de recepción de mercadería.

2. Existencia de mercadería: determinar el flujo de ingreso y salida de la misma, en este tipo de actividades es necesario destacar la importancia de su control, aquí es en donde se dan usualmente casos de robos, fraudes o manipulaciones en el control de inventarios, se deduce en un delito por parte de administradores 0 encargados del mismo. Hay que determinar la cantidad mínima y máxima de existencias que deben contener las bodegas de inventario a fin de que no exista un desabastecimiento de cierto producto o en su caso contrario, no exceda la cantidad y tenga un lugar adecuado que no permita su deterioro mientras se encuentra embodegado. Lo cual es importante conocer la cantidad de las existencias, su estado y su caducidad con el objetivo de mantener un flujo constante de artículos e insumos que entran y salen de inventarios, es aquí donde se determina el proceso de adquisición de mercadería nueva.

3. Salida de mercadería: con base en lo expuesto, la salida de las existencias del inventario deben ser controladas de manera estricta, únicamente saldrán aquellas que estén respaldadas por su correspondiente orden de despacho debidamente autorizada, donde se detalla el tipo, cantidad y destino de los artículo o insumos (Tovar, 2014).

Mantener una estructura de control interno adecuado es responsabilidad de la gerencia de la empresa o institución, las mismas que deben asegurarse de los beneficios de la implementación de una efectiva evaluación de control interno, así como de las correcciones necesarias si el caso amerita, sirve para prevenir irregularidades en el departamento de inventarios tanto con las existencias, así como con el personal que se encuentra encargado de su custodia. 


\section{MÉTODO}

La investigación fue de tipo no experimental dado que no se manipuló ninguna de las variables de estudio; el enfoque de investigación fue cuantitativo, tuvo un alcance descriptivo, pues permitió narrar las características propias de los inventarios y el control interno, así como posibilitó la explicación de todas las aristas que conciernen al manejo de los inventarios en una institución hospitalaria. El diseño de la investigación fue transversal pues se realizó en un corte de tiempo específico (Hernández, Fernández, \& Baptista, 2010).

El universo de estudio estuvo conformado por 20 jefes departamentales de las áreas operativas y administrativas del hospital Homero Castanier Crespo, relacionadas con la administración y control del inventario, utilizándose la encuesta como herramienta de recopilación de datos e información de la situación actual de la bodega e inventarios, a quienes se les aplicó encuesta mediante cuestionario tipo Likert de 17 ítems, validado por juicio de experto y cálculo de coeficiente Alfa de Cronbach de 0,86 siendo confiable para su aplicación, la información recopilada fue analizada en razón estadística descriptiva. 


\section{RESULTADOS}

Entre los principales resultados se determinó que:

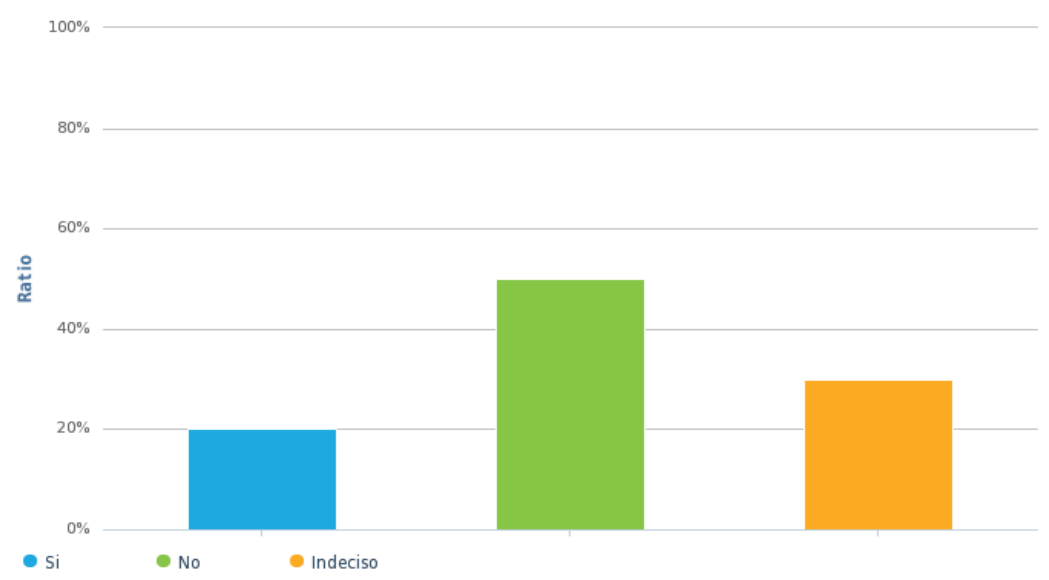

Figura 1. Cumplimiento de disposiciones de entes de control.

El $62 \%$ de encuestados que laboran en el departamento administrativo del hospital Homero Castanier Crespo, respondieron que no se realiza cumplimiento del control y seguimiento de las actividades en el departamento de bodega; un 38\% de encuestados manifestó que así ocurre. Los resultados motivan a pensar que el sistema utilizado en la actualidad, no cuenta con todas las disposiciones que vienen de parte de los organismos de control. Como se observa en la figura 1. 


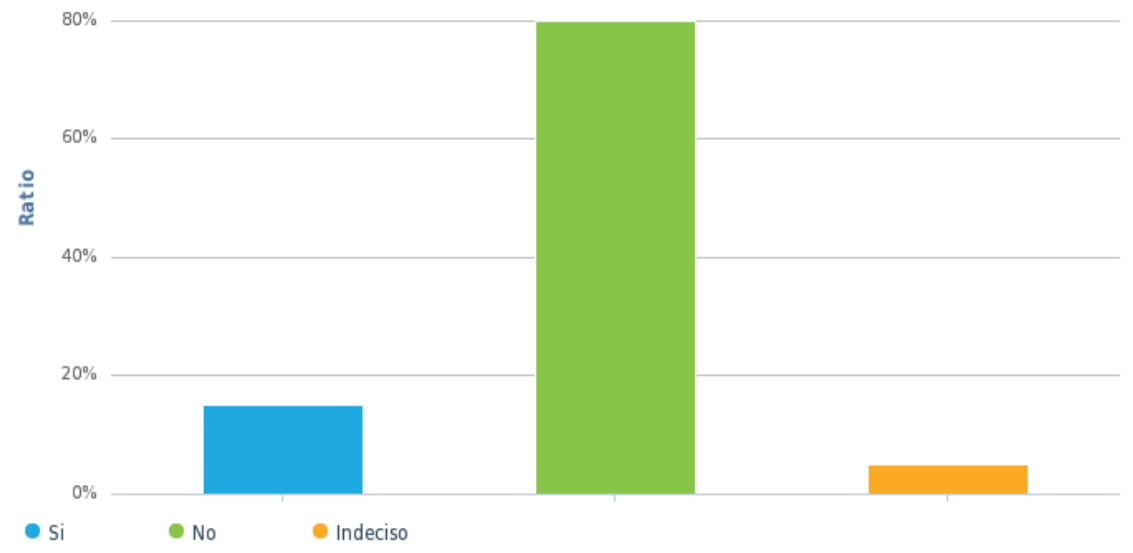

Figura 2. Espacio en bodegas.

En la figura 2 se evidencia que el $60 \%$ de los funcionarios indican que no existe el espacio en bodegas para el proceso adecuado y provoca situaciones incómodas en la atención que se realiza y retrasa la atención de los pacientes. Cabe añadir que las instalaciones físicas no siempre son las adecuadas para almacenar medicinas y equipos; al respecto, el $80 \%$ de encuestados mencionan que es una debilidad grave del departamento de bodega. Las consideraciones se agravan con la confianza que se tiene en el jefe departamental, y se observa que un $50 \%$ de los funcionarios consideran que los procesos no se llevan a cabo de forma adecuada.

A pesar de la situación negativa, el $58 \%$ de los funcionarios, señalan que la persona encargada del área tiene el perfil adecuado para el cargo, no obstante, se debe replantear la forma en que se lleva el control de los activos fijos e inventarios. Al respecto, el 50\% de los participantes respondió que el stock con relación a los intervalos de mínimos y máximos no es el adecuado, mientras que, el $40 \%$ de encuestados estableció que los inventarios no están clasificados; de manera que se observa que todavía existe una serie 
de problemas en la eficiencia del funcionamiento de bodega con relación al control de activos fijos e inventarios.

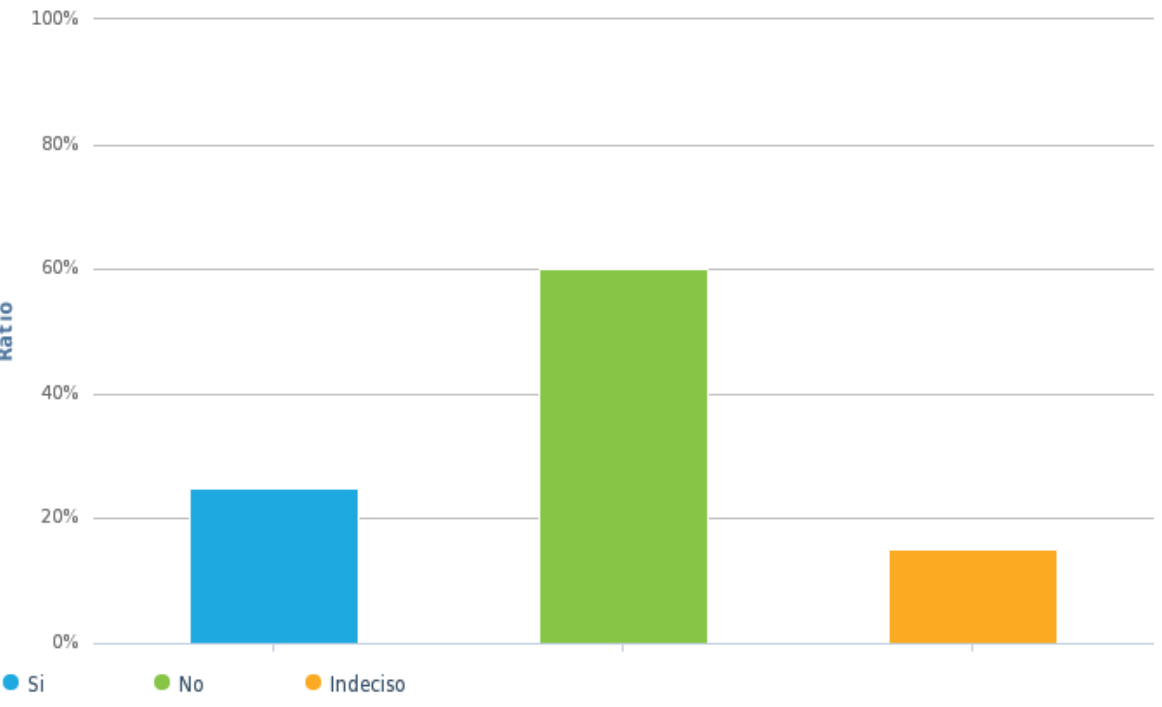

Figura 3. Eficiencia del sistema de control de inventarios.

El $40 \%$ de personal de la casa de salud, considera que el servicio brindado por este departamento es de calidad; sin embargo, se evidencia que el $60 \%$ de encuestados mencionó que el sistema para llevar a cabo el control de inventarios es ineficiente.

Los resultados muestran la necesidad de reformar el sistema con el que se desarrolla el control de los activos fijos e inventarios, diseñando un procedimiento de evaluación del sistema de control interno para considerar las fortalezas que cumple el jefe departamental, considerar el mejoramiento de eficiencia, almacenamiento y procesos. 


\section{PROPUESTA}

La propuesta señala los aspectos que deben ser considerados al realizar la evaluación de los componentes apreciados en el modelo COSO I para el control de inventarios en el Hospital Homero Castanier Crespo de la ciudad de Azogues, en aspectos que abarca la infraestructura tanto física como tecnológica, el personal, la adquisición, el almacenamiento y despacho de los insumos, equipos y materiales.

La propuesta contiene cinco fases, que abarcan todas las aristas consideradas claves en el manejo eficiente de los activos y que aportan al cumplimiento y alcance de los objetivos planteados. Previo al desarrollo de las fases a ser detalladas, se ponderan los riesgos estableciendo: riesgos bajos, moderados o altos, así como los rasgos de confianza similares para obtener información sobre el manejo adecuado de los inventarios, por medio de una matriz de riesgos.

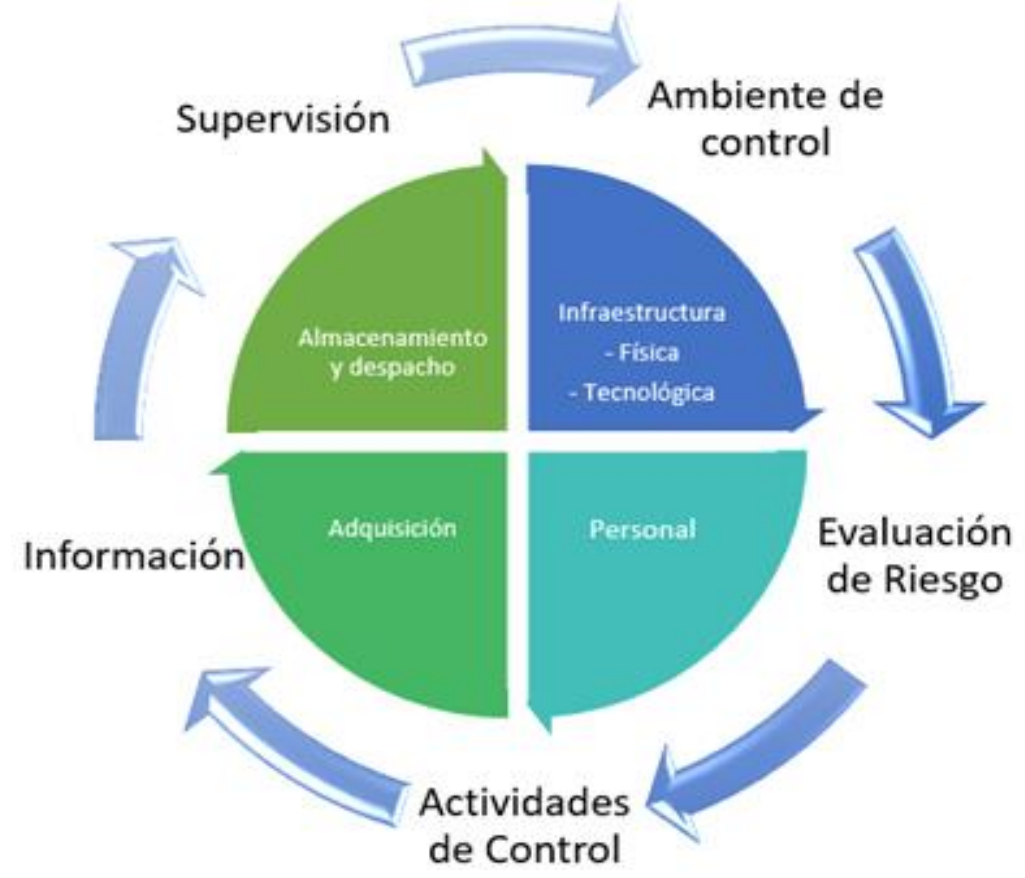

Figura 4. Propuesta de la investigación. 


\section{Fases de la propuesta}

El hospital Homero Castanier Crespo, es una entidad pública que brinda servicios de atención y tratamiento médico en distintas especialidades, ubicado en la ciudad de Azogues, provincia del Cañar. La entidad maneja suministros, equipos e insumos médicos, adquiridos mediante la aplicación de procesos determinados por la entidad de control, debiendo para ello lanzar requerimientos y elegir las mejores proformas y proveedores.

Se ha planteado como objetivo de la propuesta, establecer los aspectos considerados en el procedimiento de evaluación del sistema de control interno de inventarios bajo el modelo COSO I para el hospital Homero Castanier Crespo, con la finalidad de corregir los errores relacionados al ineficiente control que actualmente la casa de salud maneja, previa detección de los riesgos relacionados al control de inventarios.

Asimismo, su alcance, se encuentra direccionado a la evaluación de los componentes detectados en la encuesta relacionados con el sistema de control interno de inventarios que involucra la infraestructura, personal, adquisición, almacenamiento y despacho, el proceso será aplicado en el área de bodega de la Institución Pública de Salud, son los encargados del manejo de los suministros o inventarios médicos utilizados para la atención a los pacientes, considerando las directrices establecidas por el ente de control. La propuesta se fundamenta, en la necesidad de corregir los errores detectados en el manejo del sistema del control de inventario de suministros y equipos médicos. Para ello se bosquejan las actividades relacionadas a la planificación de los propósitos y la metodología del control interno.

\section{Fase I. Ambiente de control}

En la primera fase, la evaluación debe considerar las actividades de planificación en cuanto a los propósitos y la metodología del control interno que maneja la entidad, para 
María Augusta Gutiérrez-Sarmiento; Cecilia Ivonne Narváez-Zurita; Jorge Edwin Ormaza-Andrade

ello previo a la evaluación se debe contar con un claro detalle de la institución, en cuanto a sus objetivos y alcance, tiempos de trabajo, personal y diseño del programa de auditoría, detectándose todos aquellos procesos que funcionan de forma inadecuada en el departamento de bodega, el cuál es el encargado el manejo de los inventarios de la institución, debiéndose detectar los riesgos y sanear las causas y los efectos. Para ello, los elementos a fomentarse en el ambiente de control, abarcan:

Tabla 2.

Elementos del ambiente de control por componente.

\begin{tabular}{l}
\hline \multicolumn{1}{c}{ Ambiente de control } \\
\hline \multicolumn{1}{c}{ Infraestructura } \\
Sistemas tecnológicos \\
Debilidad: \\
- Sistema para el manejo de inventarios no contempla las disposiciones establecidas \\
por normativa. \\
Riesgo asociado: \\
- Sanciones administrativas, civiles y penales por la inobservancia de los lineamientos \\
$\quad$ dispuestos para el manejo, almacenamiento, adquisición y entrega del insumo, equipo, \\
material, etc. \\
Propuesta de mejora: \\
- Diseñar o adquirir un sistema adecuado y alineado a las normas establecidas. \\
- Contar con actas de recepción conforme a los requerimientos detallados, designación \\
$\quad$ de custodios, actas de entrega - recepción, actas de baja de inventarios, control de \\
existencias, etc., que respalden los procesos realizados.
\end{tabular}

\section{Layout}

Almacenamiento y despacho

\section{Debilidad:}

- Espacio inapropiado para el manejo y despacho de los insumos, equipos, instrumental y más elementos que se administran en bodega.

- Inadecuado almacenamiento (clasificación, ubicación y acceso) de medicamentos

- Ineficiente control y registro de stocks máximos y mínimos de inventarios.

\section{Riesgo asociado:}

- Retraso en el despacho de insumos, equipos, instrumental, etc., utilizados en el tratamiento de los pacientes.

- Caducidad de medicamentos e insumos que tiene fechas de vencimiento en un corto plazo.

- Pérdida de medicinas, insumos, instrumental, equipos menores, etc., generando adquisiciones innecesarias para suplir las necesidades por parte del personal médico y de otras áreas.

\section{Propuesta de mejora:}


CIENCIAMATRIA

Revista Interdisciplinaria de Humanidades, Educación, Ciencia y Tecnología

Año VI. Vol. VI. N². Edición Especial II. 2020

Hecho el depósito de ley: pp201602FA4721

ISSN-L: 2542-3029; ISSN: 2610-802X

Universidad Nacional Experimental Francisco de Miranda (UNEFM). Santa Ana de Coro. Venezuela

María Augusta Gutiérrez-Sarmiento; Cecilia Ivonne Narváez-Zurita; Jorge Edwin Ormaza-Andrade

- Diseñar un sistema adecuado y alineado a las directrices emanadas por el organismo de control, para el almacenamiento de medicinas, instrumental, equipo, etc., sustentado en un sistema informático que aporte al buen manejo de existencias.

- Rediseñar el layout del área de bodega que actualmente posee la entidad.

- Colocar mobiliario adecuado para el almacenamiento del inventario, con el fin de mejorar la visibilidad. Accesibilidad y despacho dentro en los tiempos y condiciones requeridas.

Integridad y valores éticos

Debilidad: poco interés para dar a conocer el código de ética y conducta: hace referencia a la regulación del comportamiento de las personas en el hospital Homero Castanier Crespo.

Si bien no es una coactiva el código de ética se presume una normativa interna de cumplimiento obligatorio.

Riesgo asociado: comportamiento improcedente del personal del área de inventario que afecten a las operaciones o patrimonio del hospital Homero Castanier Crespo.

Propuesta de mejora: diseñar un programa para suscitar y dar a conocer el código de ética y conducta a todos los empleados y trabajadores del hospital Homero Castanier Crespo, especia en el área de Inventarios.

\section{$>$ Compromiso de competencia profesional}

\section{Debilidades:}

- Carencia del manual de funciones administrativas y descripción de las responsabilidades individuales y colectivas en los puestos de trabajo de manera escrita.

- No existe un análisis y evaluación para que una persona ocupe un cargo en el área.

\section{Riesgos asociados}

- Inadecuada segregación de funciones, incumplimiento o mal desempeño laboral de los trabajadores.

- Mal desempeño laboral, segregación de funciones e incumplimiento por parte de los empleados.

- Posibilidad de irregularidades o errores en el proceso de contratación de personal.

\section{Propuestas de mejora}

- Preparación y entrega a los empleados de un manual que contenga los procedimientos, funciones y responsabilidades.

- Implementar un sistema de soporte de archivos de la contratación personal que contenga datos generales, tanto del perfil personal, académico y profesional de cada empleado.

\section{$>$ Administración y comité de auditoría}

Debilidades

- Inexistencia de un comité de auditoría en el hospital Homero Castanier Crespo.

- Ausencia de evaluaciones periódicas de control interno.

\section{Riesgos asociados}

- Exposición a irregularidades e ineficacias detectadas por una Auditoría Interna.

- Falta de eficiencia en el cumplimiento de metas y objetivos en el uso de los recursos compartidos e incumplimiento de las leyes.

\section{Propuestas de mejora}

- Creación de un comité de auditoría.

- Diseño de mejores procedimientos y mecanismos efectivos de control.

Departamento de talento humano: selección de personal 
CIENCIAMATRIA

Revista Interdisciplinaria de Humanidades, Educación, Ciencia y Tecnología

Año VI. Vol. VI. N². Edición Especial II. 2020

Hecho el depósito de ley: pp201602FA4721

ISSN-L: 2542-3029; ISSN: 2610-802X

Universidad Nacional Experimental Francisco de Miranda (UNEFM). Santa Ana de Coro. Venezuela

María Augusta Gutiérrez-Sarmiento; Cecilia Ivonne Narváez-Zurita; Jorge Edwin Ormaza-Andrade

\section{Debilidades:}

- Falta de procedimientos minuciosos para la selección, contratación, capacitación, estimulación, evaluación, promoción y remuneración de personal.

- Inexistencia de evaluaciones periódicas del desempeño de los empleados.

\section{Riesgos asociados}

- Contratación de recursos humanos que no tienen las habilidades y competencias aptas para cubrir un determinado puesto de trabajo.

- Problemas para asegurar y calcular el cumplimiento de las metas y objetivos propuestos.

\section{Propuestas de mejora}

- Delinear ordenamientos, cánones y políticas para la selección, contratación, capacitación, estimulación, evaluación, promoción y remuneración del personal.

- Implementar mecanismos o procedimientos de evaluación y desempeño

Debilidades:

\section{Adquisición}

- Ineficiente control y registro de stocks máximos y mínimos de inventarios.

\section{Riesgo asociado:}

- Perdida de medicinas, insumos, instrumental, equipos menores, etc., generando adquisiciones innecesarias para suplir las necesidades por parte del personal médico y de otras áreas.

Propuesta de mejora:

- Diseñar un sistema informático que aporte al buen manejo de existencias.

- Rediseñar el layout del área de bodega que actualmente posee la entidad.

- Colocar mobiliario adecuado para el almacenamiento del inventario, con el fin de mejorar la visibilidad. Accesibilidad y despacho dentro en los tiempos y condiciones requeridas, y con ello realiza eficientes procesos de adquisición y reposición del inventario, respondiendo a los cambios que el entorno presente.

- Mantener en la base de datos actualizada de estadísticas de consumo, proveedores organizados en base a su eficiencia en la entrega y calidad del producto, con la finalidad de eliminar posibles adquisiciones a proveedores de dudosa actividad.

Fuente: Elaboración propia.

\section{Fase II. Evaluación de Riesgos}

Durante la fase dos, se realiza una ponderación de riesgos, por medio de una matriz de riesgos, utilizando una hoja de cálculo para establecer riesgos bajos, moderados o altos, así como rasgos de confianza similares para obtener información sobre el manejo adecuado de los inventarios. 
CIENCIAMATRIA

Revista Interdisciplinaria de Humanidades, Educación, Ciencia y Tecnología

Año VI. Vol. VI. N². Edición Especial II. 2020

Hecho el depósito de ley: pp201602FA4721

ISSN-L: 2542-3029; ISSN: 2610-802X

Universidad Nacional Experimental Francisco de Miranda (UNEFM). Santa Ana de Coro. Venezuela

María Augusta Gutiérrez-Sarmiento; Cecilia Ivonne Narváez-Zurita; Jorge Edwin Ormaza-Andrade

Tabla 3.

Matriz de evaluación de riesgos.

\section{Evaluación de riesgo}

Área: Inventario

Factores de control: preguntas

$\mathrm{Si} \quad \mathrm{No}$ N/C $\quad$ Observaciones

\section{$>$ Objetivos globales del hospital Homero Castanier Crespo}

¿La administración ha establecido objetivos globales en el hospital Homero Castanier Crespo?

¿Los objetivos globales del hospital Homero Castanier Crespo se comunican a todos los empleados y trabajadores?

\section{$>$ Objetivos específicos}

¿Existen objetivos específicos para el manejo de inventarios?

¿Se establecen objetivos para cada actividad importante de cada proceso?

¿La administración realiza un seguimiento especial de los objetivos que forman los factores críticos de éxito?

$>$ Riesgos potenciales

¿Son identificados los riesgos potenciales para el área de Inventarios?

$>$ Gestiones para el cambio

¿Se realizan acciones que permiten el cambio en los inventarios?

¿Existe métodos para evaluar el impacto de los nuevos sistemas de inventarios?

Fuente: Elaboración propia.

La matriz de ponderación de la evaluación de control interno para el hospital Homero Castanier Crespo, determina un nivel de confianza y nivel de riesgo bajo con un rango entre $15 \%$ - 50\%, moderado $51 \%$ - $75 \%$, y alto entre $76 \%-96 \%$. 
CIENCIAMATRIA

Revista Interdisciplinaria de Humanidades, Educación, Ciencia y Tecnología

Año VI. Vol. VI. N². Edición Especial II. 2020

Hecho el depósito de ley: pp201602FA4721

ISSN-L: 2542-3029; ISSN: 2610-802X

Universidad Nacional Experimental Francisco de Miranda (UNEFM). Santa Ana de Coro. Venezuela

María Augusta Gutiérrez-Sarmiento; Cecilia Ivonne Narváez-Zurita; Jorge Edwin Ormaza-Andrade

\section{Fase III. Actividades de control}

La tercera fase mediante las pruebas de cumplimiento y pruebas sustantivas de auditoría, con técnicas de observación, análisis, inspección y cálculo, establece medidas concretas en temas de eficiencia en la entrega de la información de inventarios, el sistema que se utiliza para ingresar los datos y la organización con la que se trabaja y los insumos que se poseen, requiriéndose un flujograma del proceso renovado y con controles adecuados. El proceso deberá tener una interacción constante entre los departamentos de bodega, compras públicas y contabilidad; debiéndose para ello ejecutar las actividades de control detalladas en la tabla 4:

Tabla 4.

Actividades de control.

Actividades de control

Área: Inventario

Bodega

- El jefe del departamento de bodega receptará los pedidos y asignará los responsables de verificar el material ingresado.

- El jefe de bodega verificará que el pedido tenga la respectiva autorización.

- Si el material no cumple con los estándares especificados, se devolverán.

- Se ingresará el pedido al sistema y se almacenará los insumos y medicinas.

- Se despacharán los pedidos hospitalarios y se procederá a dar de baja el material.

- El jefe de bodega determinará la constatación física del inventario una vez por mes para compararlo con el sistema contable.

- Solo podrá ingresar a bodega el personal autorizado.

- Si existe presencia de personal no autorizado, el jefe de bodega deberá notificar a Contraloría.

$>$ Contabilidad

- El departamento de contabilidad deberá recibir, clasificar, analizar e interpretar los datos financieros emitidos por bodega.

- Preparará y realizará los informes sobre la situación financiera de los inventarios.

- Contrastará la información de la constatación física del departamento de bodega con la información financiera.

- Revisará y comparará los gastos mensuales

- Deberá mantener el archivo de documentos contables

- Comprobará las transacciones realizadas respecto a la globalidad de pedidos, autorizaciones, aprobaciones, exactitud, revisiones, cálculos y recálculos.

$>$ Compras públicas 
María Augusta Gutiérrez-Sarmiento; Cecilia Ivonne Narváez-Zurita; Jorge Edwin Ormaza-Andrade

- El departamento de compras públicas deberá recibir los pedidos emitidos desde los diversos departamentos del hospital Homero Castanier Crespo con las autorizaciones de los directivos para proceder a subir al portal de compras públicas la información.

- Se emitirán pedidos tomando en cuenta la ley y los plazos adecuados para que la recepción esté adaptada a las necesidades del hospital Homero Castanier Crespo.

- El departamento deberá mantener los contactos de los proveedores para garantizar los productos, calidad, servicio, precio y pago.

- El departamento de compras públicas deberá asegurarse que los insumos y medicinas adquiridos lleguen a bodega.

- Si no ocurre, deberá reportarlo a Contraloría.

Fuente: Elaboración propia.

La matriz de ponderación de la evaluación de control interno para el hospital Homero Castanier Crespo, determina un nivel de confianza y nivel de riesgo bajo con un rango entre $15 \%-50 \%$, moderado $51 \%-75 \%$, y alto entre $76 \%-96 \%$.

\section{Fase IV. Información y comunicación}

Desarrolla la socialización de los resultados de la evaluación de control interno con todos los miembros del hospital Homero Castanier Crespo, en los tiempos oportunos, permitiendo que el personal de la entidad capte e intercambie información relevante y requerida para desarrollar, gestionar y controlar sus operaciones. En la tabla 5 se muestra las actividades a desarrollar: 
CIENCIAMATRIA

Revista Interdisciplinaria de Humanidades, Educación, Ciencia y Tecnología

Año VI. Vol. VI. N². Edición Especial II. 2020

Hecho el depósito de ley: pp201602FA4721

ISSN-L: 2542-3029; ISSN: 2610-802X

Universidad Nacional Experimental Francisco de Miranda (UNEFM). Santa Ana de Coro. Venezuela

María Augusta Gutiérrez-Sarmiento; Cecilia Ivonne Narváez-Zurita; Jorge Edwin Ormaza-Andrade

Tabla 5.

Información y comunicación.

\begin{tabular}{|c|c|}
\hline \multicolumn{2}{|r|}{$\begin{array}{c}\text { Información y comunicación } \\
\text { Área: Inventario }\end{array}$} \\
\hline \multicolumn{2}{|r|}{ Información } \\
\hline$>$ & $\begin{array}{l}\text { Debilidad: } \\
\text { - El personal que maneja los inventarios no recibe de manera oportuna y confiable la } \\
\text { información. } \\
\text { El hospital Homero Castanier Crespo no promueve el desarrollo de un sistema de } \\
\text { inventarios moderno y acorde a las exigencias de la entidad. } \\
\text { La entidad no posee un sistema informático en el cual se descargue los insumos, } \\
\text { medicinas, máquinas, etc., requeridos y utilizados por el personal, que reporte las } \\
\text { existencias mínimas y máximas de cada bien o producto almacenado. }\end{array}$ \\
\hline$>$ & $\begin{array}{l}\text { Riesgo asociado } \\
-\quad \text { Toma de disposiciones basadas en información inexacta o no actualizada. } \\
\text { - } \quad \text { Pérdida de datos o insumos y elementos médicos en el área de Inventarios. } \\
\text { - } \quad \text { Desigualdad de datos de existencias de insumos médicos. }\end{array}$ \\
\hline$>$ & $\begin{array}{l}\text { Propuesta de mejora } \\
\text { - La información debe identificarse, procesarse y comunicarse al personal en los } \\
\text { tiempos solicitados o de ser posible en tiempo real de consulta, de tal manera que } \\
\text { puedan contar con los resultados esperados y realizar las adquisiciones en base a las } \\
\text { necesidades, sobre todo ajustadas a las realidades del entorno en un momento dado. }\end{array}$ \\
\hline \multicolumn{2}{|r|}{ Comunicación } \\
\hline$>$ & $\begin{array}{l}\text { Debilidades: inexistencia de un proceso que permita recopilar información relevante de } \\
\text { los medicamentos, equipos y suministros médicos que ingresan y salen de la bodega. } \\
\text { Riesgos asociados: pérdida de insumos, medicamentos y equipos médicos que se } \\
\text { encuentran en la bodega y a su vez generan posibles pérdidas económicas y } \\
\text { acciones legales internas y externas al personal encargado de los inventarios. } \\
\text { Propuestas de mejora } \\
\text { - Utilizar los canales de comunicación institucionales como los correos electrónicos y } \\
\text { sistemas para subir la información. } \\
\text { Prohibirse el uso de medios de comunicación personales para solicitar, confirmar o } \\
\text { enviar información de pedidos, compras, existencias de insumos o medicinas. } \\
\text { Seguir el protocolo de seguimiento formal de ingreso y egreso de los suministros } \\
\text { médicos. }\end{array}$ \\
\hline
\end{tabular}

Fuente: Elaboración propia. 
María Augusta Gutiérrez-Sarmiento; Cecilia Ivonne Narváez-Zurita; Jorge Edwin Ormaza-Andrade

La matriz de ponderación de la evaluación de control interno para el hospital Homero Castanier Crespo, determina un nivel de confianza y nivel de riesgo bajo con un rango entre $15 \%$ - 50\%, moderado $51 \%-75 \%$, y alto entre $76 \%-96 \%$.

\section{Fase V. Seguimiento}

Para finalizar en la fase cinco se corrige las falencias encontradas en el área de inventarios del hospital Homero Castanier Crespo, buscando la implementación de procesos eficientes que permitan dar un mejor servicio tanto al cliente interno como externo. Los informes deberán tener una estructura práctica y poseer los respaldos físicos y digitales, siendo necesaria la existencia de una comunicación clara, eficaz y amplia, que influya en todas las actividades, áreas y personas, debiendo de antemano comunicar claramente las responsabilidades de cada funcionario en el sistema de control interno, proporcionándose una matriz de control de las actividades requeridas para llevar a cabo el control interno en el área de inventarios.

\section{Tabla 6.}

Evaluación de riesgo.

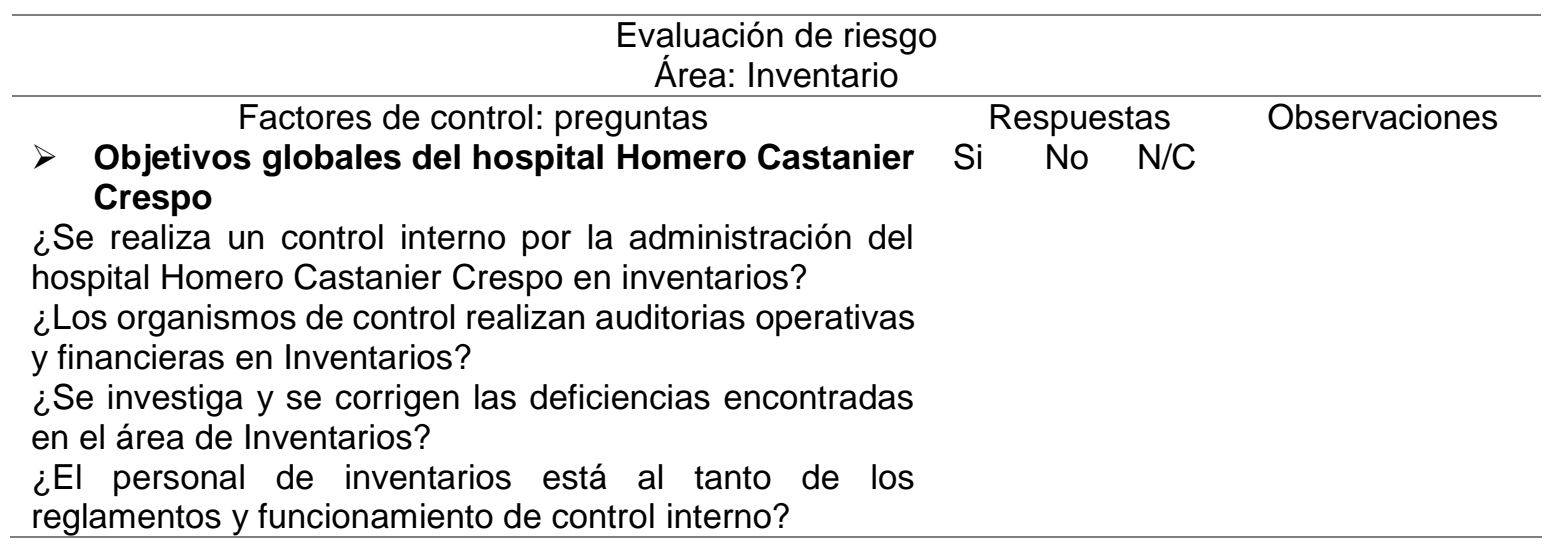


Fuente: Elaboración propia.

La matriz de ponderación de la evaluación de control interno para el hospital Homero Castanier Crespo, determina un nivel de confianza y nivel de riesgo bajo con un rango entre $15 \%-50 \%$, moderado $51 \%-75 \%$, y alto entre $76 \%-96 \%$.

\section{DISCUSIÓN}

Los sistemas de control interno permanente de inventarios exigen un mayor compromiso de la organización para involucrarse en el proceso y mantener funcionando un sistema adecuado a las necesidades de la institución hospitalaria; siendo que, los equipos médicos, los insumos y medicamentos son costosos y de empleo de los usuarios, de tal forma que, no se deben desperdiciar.

El control interno establece una relación entre las distintas funciones departamentales; al respecto, se realiza este proceso para manejar los riesgos y adoptar medidas que vayan en beneficio de la transformación de la gestión y se basa en un proceso técnico, regulado y eficaz sobre los estándares de calidad que serán reflejados en los registros contables para satisfacer demandas futuras en el uso de inventarios, así como también para la toma de decisiones asertivas en la entidad.

De la encuesta aplicada a los jefes departamentales del hospital Homero Castanier Crespo, se obtuvo que el control de inventarios con el que cuenta la institución no es el adecuado, se debería tener una interacción constante entre bodega, logística y contabilidad para llevar un control apropiado.

La percepción de los funcionarios mostró que el sistema de control no toma en cuenta la interacción que debe haber entre las diferentes áreas de la institución hospitalaria y el flujo de información no siempre presenta buenos indicadores, conduciendo todo a retrasos en la entrega de los insumos a los usuarios por el servicio obtenido. 
María Augusta Gutiérrez-Sarmiento; Cecilia Ivonne Narváez-Zurita; Jorge Edwin Ormaza-Andrade

Bajo este contexto, la aplicación de un procedimiento de evaluación del control interno de los inventarios del hospital Homero Castanier Crespo, toma en cuenta todos los elementos problemáticos y aporta con una solución para brindar una gestión efectiva que considera las fases de planificación, ejecución, informe y socialización de resultados, a través de la aplicación de fichas técnicas y ponderación de los resultados.

La propuesta planteada permitirá cumplir con la normativa nacional como el Estatuto Orgánico de Gestión Organizacional por Procesos de los Hospitales del Ministerio de Salud Pública en el que se solicita la realización del control interno y auditorías y, la disposición de información de acuerdo con los artículos 76, 81 y 88 de la Ley Orgánica de la Contraloría General del Estado.

\section{REFERENCIAS CONSULTADAS}

Ávila, Z. (2010). El Control Interno: estudio y revisión de procedimientos utilizados en la empresa Marcimex S. A. [Internal Control: study and review of procedures used in the company Marcimex S. A.]. Tesis de grado, Universidad Católica de Cuenca, Cuenca. Recuperado de https://n9.cl/zogv

Camacho Villota, W., Gil Espinoza, D., \& Paredes Tobar, J. (2017). Importancia de su funcionamiento en las empresas. [Importance of its operation in companies]. Obtenido de https://n9.cl/iknf

Cardona, J., Orejuela, J., \& Rojas, C. (2018). Gestión de inventario y almacenamiento de materias primas en el sector de alimentos concentrados. [Inventory management and storage of raw materials in the concentrated food sector]. Revista EIA, 15(30), 195-208. https://dx.doi.org/10.24050/reia.v15i30.1066

Chávez, M., \& Vallejo, C. (2017). Gestión de la información financiera y su relación con la toma de decisiones gerenciales en las organizaciones de la Unión Peruana del Norte. [Financial information management and its relationship with managerial decision making in the organizations]. Muro de la Investigación. Obtenido de https://n9.cl/0tf1e 
María Augusta Gutiérrez-Sarmiento; Cecilia Ivonne Narváez-Zurita; Jorge Edwin Ormaza-Andrade

Durán, Y. (2012). Administración del inventario: elemento clave para la optimización de las utilidades en las empresas. [Inventory management: key element for the optimization of profits in companies]. Visión Gerencial, 0(1), 55-78.

Fuertes, J. A. (2015). Métodos, técnicas y sistemas de valuación de inventarios.Un enfoque global. [Inventory Valuation Methods, Techniques and Systems: A Global Approach]. Gestión Joven(14), 48 - 65.

García, I. (2017). Economía simple. [Simple Economy]. Obtenido de https://n9.cl/1ke8

Hernández, R., Fernández, P., \& Baptista, P. (2010). Metodología de la Investigación. [Research Methodology]. McGraw - Hill Interamericana de México, S.A. de C.V.

Hurtado, L. (2014). La gerencia financiera en la toma de decisiones. [Financial management in decision making]. Bogotá: Universidad Militar Nueva Granada. Obtenido de https://n9.cl/8szrd

Juca, C., Narváez, C., Erazo, J., \& Luna, K. (2019). Modelo de gestión y control de inventarios para la determinación de niveles óptimos en la cadena de suministros de la empresa Modesto Casajoana. [Inventory management and control model for determining optimal levels in the supply chain]. 593 Digital Publisher CEIT, 4(3-1), 19-39.

López, A. A., \& Pesántez, J. (2017). Evaluación comparativa del sistema de control interno del sector comercial y del sector público del Cantón Morona. [Comparative evaluation of the internal control system of the commercial sector and the public sector of the Morona Canton]. Revista Killkana Sociales, 1(1), 31-38.

Moscoso, S. E. (2015). Propuesta de un sistema de control interno para el area de compras en la empresa logistica $L M G S$ S.A. [Proposal for an internal control system for the purchasing area in the logistics company LMG S.A]. Tesis de grado, Universidad Católica de Cuenca, Cuenca. Obtenido de https://n9.cl/x4xupy

Organización Mundial de la Salud. (2012). Introducción a la gestión de inventarios de equipo médico. [Introduction to medical equipment inventory management]. Suiza: Organización Mundial de la Salud. Obtenido de https://n9.cl/62j1

Ortega, A., Padilla, S., Torres, J., \& Ruz, A. (2017). Nivel de importancia del control interno de los inventarios dentro del marco conceptual de una empresa. [Level of 
Universidad Nacional Experimental Francisco de Miranda (UNEFM). Santa Ana de Coro. Venezuela

María Augusta Gutiérrez-Sarmiento; Cecilia Ivonne Narváez-Zurita; Jorge Edwin Ormaza-Andrade

importance of internal control of inventories within the conceptual framework of a company]. Liderazgo Estratégico, 7(1), 71-82.

Ortiz, A., Narváez, C., \& Erazo, J. (2019). Control de inventarios con enfoque ABC en el departamento de alimentos y bebidas del Hotel Oro Verde, Cuenca-Ecuador. [Inventory control with $\mathrm{ABC}$ approach in the food and beverage department of the Hotel Oro Verde, Cuenca-Ecuador]. CIENCIAMATRIA, 5(1), 735-757. https://doi.org/10.35381/cm.v5i1.316

Pazmiño, M., Narváez, C., \& Erazo, J. (2019). Herramientas para el control de inventarios inteligentes en la industria del calzado de la provincia de Tungurahua. [Tools for smart inventory control in the footwear industry in Tungurahua province]. CIENCIAMATRIA, 5(1), 758-780. https://doi.org/10.35381/cm.v5i1.318

Quinaluisa, N., Ponce, V., Muñoz, S., \& Ortega, X. P. (2018). El control interno y sus herramientas de aplicación entre COSO y COCO. [Internal control and its application tools between COSO and COCO]. Cofin Habana, 12(1), 268-283.

Rincón, C., Grajales, G., \& Zamorano, R. (2012). Estándares Internacionales en Contabilidad - ElC. [International Standards in Accounting]. Obtenido de https://n9.cl/rq8e1

Tovar, E. (2014). Auditool. Obtenido de https://n9.cl/7u34

Velasquez, S. (2011). Evaluación de los controles internos contables del área de inventario en cuanto a la recepción y despacho de la empresa Auto Repuestos Alfredo 2000 C.A., ubicada en Maracay, Estado Aragua. [Evaluation of the internal accounting controls of the inventory a]. Tesis de licenciatura, Universidad de Carabobo, La Morita. Obtenido de https://n9.cl/dmlk7

2020 por los autores. Este artículo es de acceso abierto y distribuido según los términos y condiciones de la licencia Creative Commons Atribución-NoComercial-Compartirlgual 4.0 Internacional (CC BY-NC-SA 4.0)

(https://creativecommons.org/licenses/by-nc-sa/4.0/) 